\title{
On the Development of an Exponentiated $F$ Test for One-way ANOVA in the Presence of Outlier(s)
}

\author{
Adepoju K.A*, Shittu O.I, Chukwu A.U \\ Department of Statistics, University of Ibadan, Nigeria
}

Copyright $\bigcirc 2016$ by authors, all rights reserved. Authors agree that this article remains permanently open access under the terms of the Creative Commons Attribution License 4.0 International License

\begin{abstract}
The classical Fisher-Snedecor test which compares several population means depends on the underlined assumptions which include; independent of populations, constant variance and absence of outlier among others .Arguably the source of violation of some of these assumptions is the outlier which lead to unequal variances. Outlier leads to inequality in the variances of the populations which consequently leads to the failure of the classical-F to take correct decision in terms of the null hypothesis. A series of robust tests have been carried out to ameliorate these lapses with some degrees of inaccuracies and limitations in terms of inflating the type 1 error and the power of different combination of parameters at various sample sizes while still uses the conventional F-table. This study focuses on developing robust F-test called exponentiated $\mathrm{F}$ test with the introduction of one shape parameter to the conventional F-distribution capable of taking decisions on ANOVA that are robust to the existence of outlier. The performance of the robust $\mathrm{F}$ test was compared with the existing F-tests in the literature using the power of test. Real life and simulated data were used to illustrate the applicability and efficiency of the proposed distribution over the existing ones. Experimental data with balanced and unbalanced design were used with populations sizes $\mathrm{k}=3$ and $\mathrm{k}=5$ were simulated with 10000 replications and varying degrees of outliers were ejected randomly. The results obtained indicate that the Proposed Exponentiated-F test is uniformly most powerful than the conventional-F tests for analysis of variance in the presence of outlier and is therefore recommended for use by researchers.
\end{abstract}

Keywords Fisher-Snedecor Distribution, Exponentiated-F Distribution, One Way ANOVA, Outlier, Maximum Likelihood Method

\section{Introduction}

The classical F-test to compare several population means depends on the assumption of constant variance of the population and the normality, Fisher, Ronald [11]. When these assumptions, especially the equality of variance are violated, the classical F-test lacks ability to take correction decision. There have been series of robust tests in the literature which include the Welch test [27], Scott-Smith [24], Brown-Forsythe [5], Weerahandi.s Generalized F test [26], Kenward Roger test by Heskey [17], Generalized F test[23], Parametric Bootstrap test by Krischnamoorthy [19] among others to overcome this problem but these tests still show some degrees of inaccuracies in terms of inflating the type 1 error and powers in different combinations of parameters and various sample sizes.

\subsection{One-way ANOVA Assumptions}

Considering testing the model given as

$$
\begin{gathered}
Y_{i j}=\mu+\tau_{j}+e_{i j} e_{i j} \sim N\left(\mu, \sigma^{2}\right) i=1,2, \ldots, a \\
j=1,2, \ldots, b
\end{gathered}
$$

Like any statistical test, one-way ANOVA has several assumptions. However, some of these assumptions are stringent requirements, while others can be waived.

\subsection{Outliers in Overview}

- An outlier is a data value that is in some way inconsistent with the rest of the data set.

- Outliers in the residuals- an observation for which the residual is more extreme than would be expected from random variation alone

- The identification of an outlier may change with the proposed model.

\section{Reasons for Outliers}

1) Problems with the experimental procedure

2) Errors in the recording, transcription or data input procedures

3) An incorrect or incomplete model specification

4) A genuine observation that is incompatible with the rest 


\section{Effects of removing outliers:}

- Could be negligible.

- Might affect parameter estimates.

- Might increase/decrease ResMS.

\section{- What to do?}

- Check original sources and correct erroneous values.

- Repeat analysis with the offending point(s) removed.

- Assess effect on final conclusion, parameter estimates, etc. If the results are markedly different than one may need to report both analyses.

\section{- Warning!}

- Do not discard outliers without careful consideration, as data are often expensive to obtain.

- An outlier might be the most important observation in the study.

This study focuses on developing generalized distributions that are capable of handling data that are non-normal. Also, attempts will be made to develop Robust $\mathrm{F}$-test called exponentiated $\mathrm{F}$ test that is less sensitive to any serious violations due to outliers $\$.

\subsection{What Happens If You Violate the Assumption of Equal Variances?}

For hypothesis tests like ANOVA, you set a significance level. The significance level is the probability that the test incorrectly rejects the null hypothesis (Type I error). This error causes you to incorrectly conclude that the group means are different.

If you set the significance level to 0.05 , the observed error rate is also 0.05 .

The greater the difference between the target and actual error rate, the more sensitive one-way ANOVA is to violations of the equal variances assumption.

\subsection{Possible Alternatives If Your Data Violates One-way ANOVA Assumption}

- Transformations: correcting non-normality and unequal variances by transforming all the data values

- Nonparametric tests: dealing with non-normality by employing a test that does not make the normality assumption of the one-way analysis of variance

"The Kruskal-Wallis test does not assume that the data are normally distributed; that is its big advantage. If you're using it to test whether the medians are different, it does assume that the observations in each group come from populations with the same shape of distribution, so if different groups have different shapes (one is skewed to the right and another is skewed to the left, for example, or they have different variances), the Kruskal-Wallis test may give inaccurate results "(Fagerland and Sandvik [9]).

Heteroscedasticity is one way in which different groups can have different shaped distributions. If the distributions are normally shaped but highly heteroscedastic, you can use Welch's t-test for two groups, or Welch's anova for more than two groups. If the distributions are both non-normal and highly heteroscedastic, I don't know what to recommend.

\subsection{Parametric Way Out}

The main idea of approximate tests is to possibly find a coefficient, say $m$ and degrees of freedom, say v such that

$$
F=m F^{*} \sim F(k-1, v)
$$

The main focus of the work is to develop a generalized F-Statistic for testing the equality of means when there is violation of assumptions due to outlier.

\section{Classical F Test and Existing Robust F Tests}

\subsection{F-Test /ANOVA Test of Equality of Means}

Consider b mutually stochastically independent random variables having normal distributions with unknown means $\mu_{1}, \mu_{2}, \ldots, \mu_{b}$ respectively and unknown but common variance $\sigma^{2}$. Let $X_{1 j}, X_{2 j}, \ldots, X_{a j}$ represent a random sample of size a from the normal distribution with mean $\mu_{j}$ and variance $\sigma^{2} . J=1,2, \ldots, b$.

In testing the hypothesis

$$
\begin{aligned}
& H_{0}: \mu_{1}=\mu_{2}=\ldots=\mu_{b}=\mu \\
& H_{1}: \text { not all } \mu_{\mathrm{i}} \text { 's are equal } \mathrm{i}=1, \ldots \mathrm{b}
\end{aligned}
$$

A likelihood ratio test is as follows:

The parameter $\Omega=\left\{\mu_{1}, \mu_{2}, \ldots, \mu_{b}, \sigma^{2}\right\}-\infty<\mu_{j}<\infty$,

$$
\left.0<\sigma^{2}<\infty\right)
$$

and

$$
\begin{gathered}
\omega=\left\{\mu_{1}, \mu_{2}, \ldots, \mu_{b}, \sigma^{2}\right\}-\infty<\mu_{1}=\mu_{2}=\ldots=\mu_{b}=\mu<\infty \\
\left.0<\sigma^{2}<\infty\right)
\end{gathered}
$$

The likelihood functions for $\Omega$ and $\omega$ are respectively

$$
L(\omega)=\left(\frac{1}{2 \pi \sigma^{2}}\right)^{\frac{a b}{2}} e^{-\frac{1}{2 \sigma^{2}} \sum_{j=1}^{b} \sum_{i=1}^{a}\left(X_{i j}-\mu\right)^{2}}
$$

and

$$
L(\Omega)=\left(\frac{1}{2 \pi \sigma^{2}}\right)^{\frac{a b}{2}} e^{-\frac{1}{2 \sigma^{2}} \sum_{j=1}^{b} \sum_{i=1}^{a}\left(X_{i j}-\mu_{j}\right)^{2}}
$$


The log-likelihood functions are

$\log L(\omega)=\frac{a b}{2} \log \frac{1}{2 \pi \sigma^{2}}-\frac{\sum_{j=1}^{b} \sum_{i=1}^{a}\left(X_{i j}-\mu\right)^{2}}{2 \sigma^{2}}$

$\log L(\Omega)=\frac{a b}{2} \log 2 \pi \sigma^{2}-\frac{\sum_{j=1}^{b} \sum_{i=1}^{a}\left(X_{i j}-\mu_{j}\right)^{2}}{2 \sigma^{2}}$

We then differentiate (8) and (9) with respect to $\mu, \mu_{j}$ and $\sigma^{2}$ and set the resulting expression to zero, we obtain the maximum likelihood estimate of $\mu, \mu_{j}$ and $\sigma^{2}$.

$$
\begin{aligned}
& \hat{\mu}=\bar{x}=\frac{\sum_{j=1}^{b} \sum_{i=1}^{a} X_{i j}}{a b}, \quad \hat{\sigma}^{2}=\frac{\sum_{j=1}^{b} \sum_{i=1}^{a}\left(X_{i j}-\mu\right)^{2}}{a b}, \\
& j=1,2, \ldots, b . \\
& \hat{\mu}_{j}=\bar{x}_{\cdot j}=\frac{\sum_{i=1}^{a} X_{i j}}{a} \hat{\sigma}^{2}=\frac{\sum_{j=1}^{b} \sum_{i=1}^{a}\left(X_{i j}-\bar{x}_{\cdot j}\right)^{2}}{a b}
\end{aligned}
$$

Thus, the Fisher Snedecor statistic is derived as

$$
F=\frac{\left(\sum_{j=1}^{b} \sum_{i=1}^{a}\left(X_{i j}-\bar{X}_{\cdot j}\right)^{2}\right) /(b-1)}{\sum_{j=1}^{b} \sum_{i=1}^{a}\left(X_{i j}-\bar{X}\right)^{2} /(b(a-1))}
$$

\subsection{Existing Robust Tests of Equality of Means under Violation of Assumptions}

\section{Welch's Test}

$$
\mathrm{Mw}=\left[1+\frac{2(k-2)}{\left(k^{2}-1\right)} \sum_{i=1}^{k} \frac{1}{n_{i}-1}\left(1-\frac{n_{i} / s_{i}^{2}}{\sum_{i=1}^{k} n_{i} / s_{i}^{2}}\right)^{2}\right]^{-1}
$$

and

$$
\mathrm{V}_{\mathrm{w}}=\left[\frac{3}{\left(k^{2}-1\right)} \sum_{i=1}^{k} \frac{1}{n_{i}-1}\left(1-\frac{\left(n_{i} / s_{i}^{2}\right)}{\sum_{i=1}^{k}\left(n_{i} / s_{i}^{2}\right)}\right)^{2}\right]^{-1}
$$

then

$$
\begin{aligned}
& F_{w}=M_{w} F^{*} \approx F\left(K-1, V_{w}\right) \text { and } \\
& P_{w}=P\left[F_{w}>f_{w}\right)
\end{aligned}
$$

Where $k_{i}, S_{i}$ and $n_{i}$ represent number of populations to be compared, subgroup variance and subgroup population size respectively.

\section{The Scott-Smith Test}

$$
\begin{gathered}
S_{i}^{* 2}=\frac{\left(n_{i}-1\right)}{\left(n_{i}-3\right)} S_{i}^{2} \\
\mathrm{~F}_{\mathrm{s}}=\sum_{i=1}^{k} \frac{n_{i\left(\overline{X_{i}}-\bar{X}\right)^{2}}}{S_{i}^{2 *}} \sim \chi_{R}^{2}
\end{gathered}
$$

The Brown-Forsythe Test

$$
\mathrm{B}=\frac{\sum_{i=1}^{k} n_{i}\left(\overline{X_{i}}-\bar{X}\right)^{2}}{\sum_{i=1}^{k}\left(1-\frac{n_{i}}{n}\right) S_{i}^{2}}
$$

If $\mathrm{H}_{0}$ is true, the dist of $\beta$ is $F_{K-1}, V$

$$
\mathrm{V}=\left[\sum_{i=1}^{k}\left(1-\frac{n_{i}}{n}\right) S_{i}^{2}\right]^{2} / \sum_{i=1}^{k} \frac{\left(1-\frac{n_{i}}{n}\right)^{2} S_{0}^{4}}{n_{i}-1}
$$

\section{Kenward-Roger Test}

$$
\begin{gathered}
\mathrm{a}=2 \sum_{i=1}^{k} \frac{1}{n_{i}-1}\left(1-\frac{n_{i} / S_{i}^{2}}{\sum_{i=1}^{k} \frac{n_{i}}{S_{i}^{2}}}\right)^{2} \\
\mathrm{C}_{1}=-\frac{21}{2\left(3 k^{2}+2 k+5\right)(k-1)},
\end{gathered}
$$

$$
\begin{gathered}
\mathrm{C}_{2}=\frac{7\left(k^{2}+2\right)}{2\left(3 k^{2}+2 k+5\right)(k-1)} \\
\mathrm{C}_{3}=\frac{7\left(k^{2}+2 k+4\right)}{2\left(3 k^{2}+2 k+5\right)(k-1)} E^{*}=\left(1-\frac{a}{k-1}\right)^{-1} \\
\mathrm{~V}^{*}=\frac{2}{k-1}\left(\frac{1+C_{1} a}{\left(1-\left(C_{2} a\right)\right)^{2}\left(1-\left(C_{3} a\right)\right.}\right)
\end{gathered}
$$


Denote $\ell=\frac{V^{*}}{2 E^{* 2}}$, then for the heteroscedasticity ANOVA, the Kenward-Roger approximation takes form.

$$
\begin{gathered}
\mathrm{V}_{\mathrm{KR}}=4+\frac{k+1}{(k-1) \ell-1} \text { and } \mathrm{M}_{\mathrm{KR}}=\frac{V_{K R}}{E^{*}\left(V_{K R}-2\right)} \\
F_{K R}=M_{K R} F^{*} \approx F\left(k-1, V_{K R}\right) \\
P_{K R}=P\left(F_{K R}>f_{K R}\right)
\end{gathered}
$$

\section{Parametric Bootstrap Test}

This allows resampling from a distribution under $\mathrm{H}_{0}$ whose parameters are the sample variances.

Generate independent $Z_{i} \sim \mu(0,1)$ and $\chi_{n i-1}^{2}$ random variable, we set

$$
\begin{aligned}
& \overline{Y_{\beta i}}=\sqrt{\frac{S_{i}^{2}}{n_{i}}} Z_{i} \text { and } \\
& S_{\beta i}^{2}=\frac{S_{i}^{2}}{n_{i}-1} \chi_{n_{i}-1,}^{2} i=1,2, \ldots, k \\
& F_{P \beta}=\frac{1}{K-1}\left[\sum_{i=1}^{k}\left(\frac{n_{i}}{S_{\beta i}^{2}}\right) \bar{Y}_{\beta_{i i}}^{2}-\left[\sum_{i=1}^{k} \frac{\left(\frac{n_{i}}{S_{\beta i}^{2}}\right) \bar{Y}_{\beta_{i}}}{\sum_{i=1}^{k} \frac{n_{i}}{S_{\beta i}^{2}}}\right]^{2}\right] \\
& \chi^{2} S_{b \beta}^{2}=\sum_{i=1}^{K} \frac{Z_{1}^{2}\left(n_{i}-1\right)}{\chi^{2} n_{i}=1}-\frac{\left[\sum_{i=1}^{k} \frac{\sqrt{n_{i}}}{S_{i} \chi_{n_{i}-1}^{2}}\left(n_{i}-1\right)\right]^{2}}{\sum_{i=1}^{k} n_{i}\left(n_{i}-1\right) S_{i} \chi_{n_{i}-1}^{2}}
\end{aligned}
$$

\subsection{Proposed Exponentiated F-Distribution and F-Tests}

Let $\mathrm{X}$ be a random variable with $\mathrm{F}$-distribution, its density and distribution functions are respectively defined as

$$
\begin{gathered}
f(x)=\frac{r_{1}^{\frac{r_{1}}{2}} x^{\frac{r_{1}}{2}-1}}{\beta\left(\frac{r_{1}}{2}, \frac{r_{2}}{2}\right) r_{2}^{\frac{r_{1}}{2}}\left(1+\frac{r_{1} x}{r_{2}}\right)^{\frac{r_{1}+r_{2}}{2}}}, \\
x>0, r_{1}>0, r_{2}>0 \\
F(x)=A=I_{\frac{r 1 x}{r 2}}=\frac{\beta\left(\frac{r_{1} x}{r_{2}} ; \frac{r_{1}}{2}, \frac{r_{2}}{2}\right)}{\beta\left(\frac{r_{1}}{2}, \frac{r_{2}}{2}\right)}
\end{gathered}
$$

Using the function previously used by Nadarajah and Kotz [21], called the exponentiated link function as follows

$$
g(x)=c[F(x)]^{c-1} f(x)
$$

If we put (12) and (13) in (14), we have the proposed exponentiated-F distribution as

$$
g(x)=\frac{c I_{\frac{r_{1} x}{r_{2}}}^{c-1} r_{1}^{\frac{r_{1}}{2}} x^{\frac{r_{1}-1}{2}}}{\beta\left(\frac{r_{1}}{2}, \frac{r_{2}}{2}\right) r_{2}^{\frac{r_{1}}{2}}\left(1+\frac{r_{1} x}{r_{2}}\right)^{\frac{r_{1}+r_{2}}{2}}}
$$

As for the proposed exponentiated $\mathrm{F}$ test, it is derived as follows

$$
\begin{gathered}
f(x)=\frac{1}{\sqrt{2 \pi \sigma^{2}}} e^{-\frac{1}{2}\left(\frac{x-\mu}{\sigma}\right)^{2}} \\
F(x)=\frac{\gamma\left[\left(\frac{x-\mu}{\sigma}\right)^{2}, \frac{1}{2}\right]}{\Gamma\left(\frac{1}{2}\right)}=P\left[\left(\frac{x-\mu}{\sigma}\right)^{2}, \frac{1}{2}\right]=\Phi\left(\left(\frac{x-\mu}{\sigma}\right)\right)
\end{gathered}
$$

Firstly, we obtain exponentiated normal distribution found using (16) and (17) in (14)

$$
\begin{gathered}
g(x)=c P\left[\left(\frac{x-\mu}{\sigma}\right)^{2}, \frac{1}{2}\right]^{C-1} \frac{1}{\sqrt{2 \pi \sigma^{2}}} e^{-\frac{1}{2}\left(\frac{x-\mu}{\sigma}\right)^{2}} \\
x>0, \mu>0, \sigma>0, c>0
\end{gathered}
$$

$L\left(\omega_{1}\right)=$

$C^{a b} \sum_{j=1}^{b} \sum_{i=1}^{a} P\left[\left(\frac{x_{i j}-\mu}{\sigma}\right)^{2}, \frac{1}{2}\right]^{C-1}\left(\frac{1}{\sqrt{2 \pi \sigma^{2}}}\right)^{\frac{a b}{2}} e^{-\frac{1}{2 \sigma^{2}}} \sum_{j=1}^{b} \sum_{i=1}^{a}\left(X_{i j}-\mu\right)^{2}$ $L\left(\Omega_{1}\right)=$

$C^{a b} \sum_{j=1}^{b} \sum_{i=1}^{a} P\left[\left(\frac{x_{i j}-\mu}{\sigma}\right)^{2} \frac{1}{2}\right]^{C-1}\left(\frac{1}{\sqrt{2 \pi \sigma^{2}}}\right)^{\frac{a b}{2}} e^{-\frac{1}{2 \sigma^{2}} \sum_{j=1}^{b} \sum_{i=1}^{a}\left(x_{i j}-\mu_{j}\right)^{2}}$

Taking the $\log$ of likelihood functions in (19) and differentiating with respect to $\mu$ and $\sigma$, we have

$$
\begin{gathered}
\bar{x}=\frac{\sum_{j=1}^{b} \sum_{i=1}^{a}\left(x_{i j}\right)}{a b}-\frac{2(c-1) \sigma}{\sqrt{\pi}} \\
\hat{\sigma}^{2}=\frac{\sum_{j=1}^{b} \sum_{i=1}^{a}\left(x_{i j}-\bar{x}_{\bullet j}\right)^{2}+2(c-1) \sum_{j=1}^{b} \sum_{i=1}^{a}\left(x_{i j}-\bar{x}_{\bullet j}\right)^{3}}{a b}
\end{gathered}
$$




$$
\hat{C}_{\omega_{1}}=\frac{-a b}{\sum_{j=1}^{b} \sum_{i=1}^{a} \log P\left[\frac{1}{2},\left(\frac{x_{i j}-\bar{x}}{\sigma}\right)^{2}\right]}
$$

The proposed exponentiated F-statistic is

$$
F=\frac{\left(2 \pi a \sum_{j=1}^{b}\left(\bar{x}_{\bullet j}-\bar{x}\right)^{2}+\frac{2(c-1)}{\sigma \sqrt{\pi}} \sum_{j=1}^{b} \sum_{i=1}^{a}\left(x_{i j}-\bar{x}\right)^{2}+\right) /(b-1)}{2 \pi \sum_{j=1}^{b} \sum_{i=1}^{a}\left(x_{i j}-\bar{x}_{\bullet j}\right)^{2}+\frac{2(c-1)}{\sigma \sqrt{\pi}} \sum_{j=1}^{b} \sum_{i=1}^{a}\left(x_{i j}-\bar{x}_{\bullet j}\right)^{3} / b(a-1)}
$$

\section{Applications}

\begin{tabular}{|c|c|c|c|c|c|c|c|c|}
\hline ni & $\sigma_{i}^{2}$ & C F (1925) & $\begin{array}{c}\text { WT } \\
(1951)\end{array}$ & SS (1971) & $\begin{array}{c}\text { BF } \\
(1974)\end{array}$ & $\begin{array}{c}\text { KR } \\
(1992)\end{array}$ & $\begin{array}{c}\text { PB } \\
(2007)\end{array}$ & $\begin{array}{l}\text { PROPOSD } \\
\text { EXOP-F }\end{array}$ \\
\hline \multirow{8}{*}{$3,3,3$} & $1,1,1$ & 0.0526 & 0.0466 & NA & 0.0403 & 0.0203 & 0.0400 & 0.0039 \\
\hline & $100,100,100$ & 0.0493 & 0.0226 & NA & 0.0491 & 0.0130 & 0.0405 & 0.0031 \\
\hline & $1,100,100$ & 0.0777 & 0.0741 & NA & 0.0471 & 0.0000 & 0.0076 & 0.0015 \\
\hline & $100,1,100$ & 0.0772 & 0.0675 & NA & 0.0484 & 0.0000 & 0.0070 & 0.0000 \\
\hline & $100,100,1$ & 0.0734 & 0.0720 & NA & 0.0136 & 0.0000 & 0.0064 & 0.0008 \\
\hline & $100,1,1$ & 0.1467 & 0.0472 & NA & 0.0464 & 0.0000 & 0.0575 & 0.0116 \\
\hline & $1,100,1$ & 0.1545 & 0.0536 & NA & 0.0505 & 0.0303 & 0.0576 & 0.0109 \\
\hline & $1,1,100$ & 0.0225 & 0.0648 & NA & 0.0507 & 0.0294 & 0.0584 & 0.0118 \\
\hline
\end{tabular}

Table 1. Simulated Type I Error Rates When $K=3$ For Equal Sample Sizes $(3,3,3)$

Table 2. Simulated Type I Error Rates When $\mathrm{K}=3$ For Equal Sample Sizes $(5,5,5)$

\begin{tabular}{|c|c|c|c|c|c|c|c|c|}
\hline \multirow{n}{*}{$\mathrm{ni}$} & $\sigma_{i}^{2}$ & $\mathrm{CF}(1925)$ & $\mathrm{WT}(1951)$ & $\mathrm{SS}(1971$ & $\begin{array}{c}\text { BF } \\
(1974)\end{array}$ & $\begin{array}{c}\text { KR } \\
(1992)\end{array}$ & $\begin{array}{c}\text { PB } \\
(2007)\end{array}$ & $\begin{array}{c}\text { PROPOSED } \\
\text { EXPO-F }\end{array}$ \\
\hline \multirow{5}{*}{$5,5,5$} & $1,1,1$ & 0.0514 & 0.0448 & 0.0398 & 0.0489 & 0.0446 & 0.0767 & 0.0000 \\
\cline { 2 - 10 } & $100,100,100$ & 0.0502 & 0.0453 & 0.0339 & 0.0486 & 0.0379 & 0.0557 & 0.0032 \\
\cline { 2 - 10 } & $1,100,100$ & 0.0737 & 0.0601 & 0.9434 & 0.0505 & 0.0451 & 0.1002 & 0.0036 \\
\cline { 2 - 10 } & $100,1,100$ & 0.0740 & 0.0612 & 0.9416 & 0.0516 & 0.0476 & 0.1010 & 0.0031 \\
\cline { 2 - 10 } & $100,100,1$ & 0.0719 & 0.0530 & 0.9405 & 0.0524 & 0.0510 & 0.1055 & 0.0028 \\
\cline { 2 - 10 } & $100,1,1$ & 0.1254 & 0.0574 & 0.9389 & 0.0586 & 0.0510 & 0.0886 & 0.0500 \\
\cline { 2 - 10 } & $1,100,1$ & 0.1210 & 0.0559 & 0.9457 & 0.0549 & 0.0476 & 0.0813 & 0.0495 \\
\hline
\end{tabular}

Table 3. Simulated Type I Error Rates When $\mathrm{K}=3$ For Equal Sample Sizes $(7,7,7)$

\begin{tabular}{|c|c|c|c|c|c|c|c|c|}
\hline \multirow{2}{*}{$\mathrm{ni}$} & $\sigma_{i}^{2}$ & $\mathrm{CF}(1925)$ & $\mathrm{WT}(1951)$ & $\begin{array}{c}\mathrm{SS} \\
(1971)\end{array}$ & BF (1974) & KR (1992) & PB $(2007)$ & $\begin{array}{c}\text { PROPOSED } \\
\text { EXPO-F }\end{array}$ \\
\hline \multirow{7}{*}{$7,7,7$} & $1,1,1$ & 0.0481 & 0.0430 & 0.0296 & 0.0440 & 0.0454 & 0.0764 & 0.0014 \\
\cline { 2 - 10 } & $100,100,100$ & 0.0499 & 0.0505 & 0.0327 & 0.0480 & 0.0475 & 0.0679 & 0.0026 \\
\cline { 2 - 10 } & $1,100,100$ & 0.0651 & 0.0516 & 0.9482 & 0.0434 & 0.0439 & 0.0835 & 0.0031 \\
\cline { 2 - 10 } & $100,1,100$ & 0.0666 & 0.0521 & 0.9450 & 0.0550 & 0.0485 & 0.0914 & 0.0032 \\
\cline { 2 - 10 } & $100,100,1$ & 0.0644 & 0.0552 & 0.9459 & 0.0547 & 0.0446 & 0.0825 & 0.0082 \\
\cline { 2 - 10 } & $100,1,1$ & 0.1029 & 0.0575 & 0.9533 & 0.0677 & 0.0486 & 0.0782 & 0.0544 \\
\cline { 2 - 10 } & $1,100,1$ & 0.1040 & 0.0481 & 0.9516 & 0.0644 & 0.0461 & 0.0727 & 0.0488 \\
\hline
\end{tabular}


Table 4. Simulated Type I Error Rates When $\mathrm{K}=3$ For Equal Sample Sizes $(10,10,10)$

\begin{tabular}{|c|c|c|c|c|c|c|c|c|}
\hline $\mathrm{ni}$ & $\sigma_{i}^{2}$ & $\mathrm{CF}(1925)$ & $\mathrm{WT}(1951)$ & $\mathrm{SS}(1971$ & $\mathrm{BF}(1974)$ & $\mathrm{KR}(1992)$ & $\mathrm{PB}(2007)$ & EXPO-F \\
\hline \multirow{5}{*}{$10,10,10$} & $1,1,1$ & 0.0479 & 0.0497 & 0.0319 & 0.0479 & 0.0474 & 0.0625 & 0.0007 \\
\cline { 2 - 10 } & $100,100,100$ & 0.0497 & 0.0503 & 0.0304 & 0.0513 & 0.0480 & 0.0652 & 0.0008 \\
\cline { 2 - 10 } & $1,100,100$ & 0.1033 & 0.0498 & 0.9517 & 0.0558 & 0.0465 & 0.0790 & 0.0013 \\
\cline { 2 - 10 } & $100,1,100$ & 0.0691 & 0.0533 & 0.9515 & 0.0529 & 0.0472 & 0.0785 & 0.0041 \\
\cline { 2 - 10 } & $100,100,1$ & 0.0966 & 0.0535 & 0.9535 & 0.0519 & 0.0484 & 0.0797 & 0.0023 \\
\cline { 2 - 10 } & $100,1,1$ & 0.1420 & 0.0532 & 0.9582 & 0.0728 & 0.0500 & 0.0710 & 0.0440 \\
\cline { 2 - 10 } & $1,100,1$ & 0.0998 & 0.0526 & 0.9554 & 0.0664 & 0.0490 & 0.0652 & 0.0444 \\
\hline
\end{tabular}

Table 5. Simulated Type Error Rates When $\mathrm{K}=5$ and Equal Sample Sizes

\begin{tabular}{|c|c|c|c|c|c|c|c|c|}
\hline $\mathrm{ni}$ & $\sigma_{i}^{2}$ & $\begin{array}{c}\mathrm{CF} \\
(1925)\end{array}$ & $\begin{array}{c}\text { WT } \\
(1951)\end{array}$ & SS (1971 & $\begin{array}{c}\mathrm{BF} \\
(1974)\end{array}$ & KR (1992) & PB (2007) & EXPO \\
\hline \multirow{3}{*}{$3,3,3,3,3$} & $100,100,100,100,100$ & 0.0527 & 0.0663 & NA & 0.0142 & NA & 0.0592 & 0.0069 \\
\hline & $100,100,100,100,1$ & 0.0683 & 0.1191 & NA & 0.0275 & NA & 0.0001 & 0.0134 \\
\hline & $100,1,1,1,1$ & 0.2009 & 0.0741 & NA & 0.0453 & NA & 0.0581 & 0.0157 \\
\hline \multirow{3}{*}{$5,5,5,5,5$} & $1,1,1,1,100$ & 0.1717 & 0.0605 & 0.9308 & 0.0649 & 0.0398 & 0.0939 & 0.0090 \\
\hline & $1,100,100,100,100$ & 0.0631 & 0.0741 & 0.9291 & 0.0445 & 0.0454 & 0.1188 & 0.0131 \\
\hline & $100,100,100,100,100$ & 0.0500 & 0.0507 & 0.0530 & 0.0363 & NA & 0.0664 & 0.0147 \\
\hline \multirow{3}{*}{$7,7,7,7,7$} & $100,100,100,100,100$ & 0.0529 & 0.0506 & 0.0502 & 0.0404 & 0.0371 & 0.0697 & 0.0213 \\
\hline & $100,100,1,100,100$ & 0.0646 & 0.0665 & 0.9376 & 0.0582 & 0.0472 & 0.0957 & 0.0189 \\
\hline & $1,1,1,1,100$ & 0.1527 & 0.0553 & 0.9384 & 0.0815 & 0.0388 & 0.0789 & 0.0163 \\
\hline
\end{tabular}

Table 6. Simulated Type 1 Error Rates When $\mathrm{K}=3$ and Unequal Sample Sizes

\begin{tabular}{|c|c|c|c|c|c|c|c|c|}
\hline ni & $\sigma_{i}^{2}$ & $\begin{array}{c}C \text { C F } \\
(1925)\end{array}$ & $\begin{array}{c}\text { WT } \\
(1951)\end{array}$ & SS (1971) & BF (1974) & KR (1992) & $\begin{array}{c}\text { PB } \\
(2007)\end{array}$ & $\begin{array}{l}\text { PROPOSED } \\
\text { EXPO-F }\end{array}$ \\
\hline \multirow{3}{*}{$2,3,3$} & $100,100,100$ & 0.0461 & 0.1453 & NA & 0.0335 & 0.0375 & 0.0477 & 0.0165 \\
\hline & $100,100,1$ & 0.0118 & 0.0728 & NA & 0.0324 & 0.7025 & 0.8714 & 0.0485 \\
\hline & $100,1,1$ & 0.3190 & 0.1108 & NA & 0.0450 & 0.0918 & 0.1029 & 0.1571 \\
\hline \multirow{3}{*}{$3,5,7$, } & $100,100,100$ & 0.0518 & 0.2899 & NA & 0.0363 & 0.0317 & 0.0921 & 0.0000 \\
\hline & $100,100,1$ & 0.1476 & 0.2290 & NA & 0.0402 & 0.0491 & 0.1128 & 0.0002 \\
\hline & $100,1,1$ & 0.3357 & 0.2540 & NA & 0.0498 & 0.0541 & 0.1167 & 0.0449 \\
\hline \multirow{3}{*}{$5,7,10$} & $100,100,100$ & 0.0485 & 0.3470 & 0.9531 & 0.0446 & 0.0426 & 0.0766 & 0.0000 \\
\hline & $100,100,1$ & 0.1273 & 0.3138 & 0.9561 & 0.0463 & 0.0554 & 0.1098 & 0.0000 \\
\hline & $100,1,1$ & 0.2322 & 0.3354 & 0.9472 & 0.0544 & 0.0513 & 0.0946 & 0.0013 \\
\hline \multirow{3}{*}{$4,8,15$} & $100,100,100$ & 0.0507 & 0.3514 & 0.0306 & 0.0374 & 0.0487 & 0.0895 & 0.0000 \\
\hline & $100,100,1$ & 0.2134 & 0.3009 & 0.9578 & 0.0330 & 00.513 & 0.01210 & 0.0000 \\
\hline & $100,1,1$ & 0.3864 & 0.3203 & 0.9355 & 0.0536 & 0.0548 & 0.1148 & 0.0063 \\
\hline
\end{tabular}


Table 7. Simulated Type 1 Error Rates When $\mathrm{K}=5$ and Unequal Sample Sizes

\begin{tabular}{|c|c|c|c|c|c|c|c|c|}
\hline $\mathrm{ni}$ & $\sigma_{i}^{2}$ & $\begin{array}{c}\text { CF } \\
(1925) \\
\end{array}$ & $\begin{array}{c}\text { WT } \\
(1951) \\
\end{array}$ & $\begin{array}{c}\text { SS } \\
(1971 \\
\end{array}$ & $\begin{array}{c}\text { BF } \\
(1974) \\
\end{array}$ & $\begin{array}{c}\text { KR } \\
(1992) \\
\end{array}$ & $\begin{array}{c}\text { PB } \\
(2007) \\
\end{array}$ & $\begin{array}{c}\text { PROPOSEE } \\
\text { XP-F } \\
\end{array}$ \\
\hline \multirow{3}{*}{$2,2,3,3,5$} & $100,100,100,100,100$ & 0.0514 & 0.0112 & NA & 0.0559 & 0.0000 & 0.0151 & 0.0000 \\
\hline & $100,100,100,100,1$ & 0.1486 & 0.0273 & NA & 0.0044 & 0.0000 & 0.0125 & 0.0000 \\
\hline & $100,1,1,1,1$ & 0.4256 & 0.3258 & NA & 0.0384 & 0.0046 & 0.0106 & 0.0455 \\
\hline \multirow{3}{*}{$4,4,6,6,10$} & $100,100,100,100,100$ & 0.0488 & 0.0308 & 0.0419 & 0.0505 & 0.0244 & 0.0645 & 0.0000 \\
\hline & $100,100,100,100,1$ & 0.1258 & 0.0671 & 0.9276 & 0.442 & 0.0001 & 0.0001 & 0.0000 \\
\hline & $100,1,1,1,1$ & 0.2999 & 0.2703 & 0.9166 & 0.0541 & 0.0000 & 0.0000 & 0.0032 \\
\hline \multirow{3}{*}{$3,5,7,10,10$} & $100,100,100,100,100$ & 0.0509 & 0.0191 & 0.0258 & 0.0504 & 0.0387 & 0.0769 & 0.0000 \\
\hline & $100,100,100,100,1$ & 0.1010 & 0.0428 & 0.9329 & 0.0457 & 0.0000 & 0.0000 & 0.0000 \\
\hline & $100,1,1,1,1$ & 0.4692 & 0.4328 & 0.9082 & 0.0505 & 0.0002 & 0.0002 & 0.0158 \\
\hline
\end{tabular}

Adepoju,

\section{Conclusions}

The available tests for the one-way ANOVA model with heteroscedastic error variances haveserious type I error problems that have been overlooked; this has been pointed out by Dajani [7]. In this paper, we have developed the Exponentiated-F test as an alternative to Classical $F$ test and compared to some of the existing Robust $\mathrm{F}$ tests in the literature which include: the Welch test [27], the Scot-Smith test [24], the Brown-Forsythe test [5], the Kenward Roger test [17] and Parametric Bootstrapping test by Krischnamoorthy et al [19].

For a range of choices of the sample size and parameter configurations, we have investigated the performance of the above tests using Monte Carlo simulation. In terms of controlling the Type I error rate, one can conclude in strong term that the Proposed Exponentiated-F test is the only procedure that performs satisfactorily, regardless of the sample sizes, values of the error variances, and the number of means being compared both in the presence and absence of outlier. The Kenward-Roger test came a distant second.

\section{REFERENCES}

[1] Adepoju, K. A, Chukwu A. U and Shittu, O.I (2014)"The Statistical properties of exponentiated Nakagami Distribution. (International Journal of Mathematics and System, vol 7, 2014)

[2] Akinsete, A. and Lowe,(2008).The beta-Rayleigh distribution in reliability measure. Section on Physical and Engineering Sciences, Proceedings of the American Statistical Association, (1): $3103-3107$.

[3] Akinsete, A., Famoye, F. and Lee, C. (2008) the beta-Pareto distribution, Statistics, 42(6): 547-563.

[4] Barnard, G. A. (1984).Comparing the means of two independent samples. Applied Statistics 33, 266-271.

[5] Brown, M.B. - Forsythe A.B. (1974). The small sample behavior of some statistics which test the equality of several means, Technometrics 16, 1974, 129-132
[6] Chukwu A. U., Adepoju, K. A (2012): "On the Power Efficiency of Artificial Neural Network (ANN) and the Classical Regression Model". ( Canada journal of progress in applied Mathematics, vol 3, No2, 2012)

[7] Dajani, A. N., 2002. Contributions to Statistical Inference for Some Fixed and Random Models. Ph.D. Dissertation submitted to the Department of Mathematics and Statistics, University of Maryland - Baltimore County.

[8] Eugene, N., Lee, C. and Famoye, (2002) F. Beta-normal distribution and its applications. Communications in Statistics-theory and Methods, 31(4):497-512.

[9] Fagerland MW, Sandvik L. (2009) The Wilcoxon-Mann-Whi tney tests under scrutiny. Stat Med.2009; 28:1487-1497

[10] Famoye, F., Lee, C. and Olugbenga, O (2005). The Beta-Weibull distribution, Journal of Statistical Theory and Applications, 4(2):121-138.

[11] Fisher, Ronald (1918). "Studies in Crop Variation. I. An examination of the yield of dressed grain from Broadbalk" . Journal of Agricultural Science 11: 107-135.

[12] Hastings, W.K. (1979) Monte Carlo sampling methods using Markov chains and their applications. Biometrika, 57:97-109.

[13] Hampel, F. R., Ronchetti, E. Z., Rousseeuw, P. J. and Stahel, W.A. (1986). Robust Statistics. The approach based on influence functions. New York: Wiley.

[14] James, G.S., (1951). The comparison of several groups of observations when the ratios of population variances are unknown. Biometrika 38, 324-329

[15] Jones M. C.(2004) Families of distributions arising from distributions order statistics. Sociedad de Estadistica e Investigacion Operativa Test, 13(1):1-43.

[16] Kozubowski, T. and Nadarajah S (2008). The beta-Laplace distribution. Journal of computational analysis and Applications, 10(3): 305-318.

[17] Kenward, M. G.-Roger, J. H. Small Sample Inference for Fixed Effects from Restricted Maximum Likelihood, Biometrics 53, 1997, 983-997.

[18] Krutchkoff, R. G. 1988. One-way fixed effects analysis of variance when the error variances may be unequal. Journal of Statistical Computation and Simulation 30, 259-271 
[19] Krischnamoorthy, K., Lu, Fei, and Mathew, T. A parametric bootstrap approach for ANOVA with unequal variances: Fixed and random models, Computational Statistics \& Data Analysis51, 2007, 5731-5742.

[20] Li, B.W., Cardozo, M.S., (1994). Determination of total dietary fiber in foods or products with little or no starch, non-enzymatic-gravimetric method: Collaborative study. J. Assoc. Analytical Chemists International 77, 687-689.

[21] Nadarajah, S. and Kotz, S (2006).The beta-exponential distribution. Reliability Engineering and System Safety, 91(1):689-697.

[22] Nadarajah S. \& Kotz S. (2006). "The Exponentiated Type Distributions". Acta Applicandae Mathematica. 92 (2). 97-111.
[23] Rice, W.R and Gaines, S.D. (1989). One-way analysis of variance with unequal variances. Proceedings of the National Academy of Sciences 86, 8183-8184

[24] Scott, A. J. ve Smith, T. M. F., Interval Estimates for Linear Combinations of Means, Applied Statistics, 20:276-285 (1971)

[25] Tsui, K. and Weerahandi, S. (1989).Generalized p-values in significance testing of hypotheses in the presence of nuisance parameters. Journal of the American Statistical Association 84, 602-607.

[26] Weerahandi.s, S. (1987).Testing regression equality with unequal variances. Econometrica 55, 1211-1215.

[27] Welch, B.L., (1951). On the comparison of several mean values: An alternative approach. Biometrika 38, 330-336. 